\title{
Intolerancia y miopatía por estatinas
}

\author{
Statin intolerance and myopathy
}

\author{
Raúl Ariza Andraca, ${ }^{*}$ José Halabe Cherem, ${ }^{\ddagger}$ Alberto Lifshitz Guinzberg*
}

\section{Resumen}

Los efectos adversos que se presentan con más frecuencia por el empleo de estatinas son los musculoesqueléticos. Sus manifestaciones van desde el efecto nocebo, mialgias, debilidad, atrofia muscular, miositis, y, excepcionalmente, rabdomiólisis. Su patogenia es diversa, pero destacan la predisposición individual y la multimorbilidad. Existen estrategias para su tratamiento y prevención; para los casos de intolerancia verdadera o de efectos tóxicos graves, ya existen medicamentos alternativos y eficaces para disminuir el c-LDL. Las estatinas representan uno de los medicamentos más empleados en la terapéutica médica actual, por lo que es importante conocer y tener presente sus efectos adversos para su prevención, vigilancia, detección y tratamiento oportuno.

Palabras clave: Miopatía por estatinas, toxicidad por estatinas, efectos adversos de hipolipemiantes, miopatía por fármacos, intolerancia a estatinas.

Usted está enfermo de medicina... El doctor inverosímil, Ramón Gómez de la Serna

Las estatinas son los medicamentos más eficaces y los que mayormente se emplean para el tratamiento de la hipercolesterolemia. Éstas se incluyeron en la práctica médica desde 1987 y son consideradas como los fármacos más efectivos para reducir la lipoproteína de baja densidad (c-LDL). Su administración por tiempo prolongado ha logrado disminuir la morbilidad y mortalidad cardiovasculares. ${ }^{1,2}$

\footnotetext{
* División de Postgrado.

‡ Jefe de la División de Postgrado.
}

Facultad de Medicina de la Universidad Nacional Autónoma de México (UNAM). Ciudad de México, México.

Correspondencia:

Dr. Raúl Ariza Andraca

Correo electrónico: craulariza@yahoo.com.mx

Aceptado: 15-06-2020.

www.medigraphic.com/actamedica

\section{Abstract}

Adverse events due to statin's administration are mainly confined to the musculoskeletal system. The different manifestations include nocebo effects, myalgias, muscular atrophy, myositis, and rarely rhabdomyolisis. Several etiopathogenic mechanisms are involved, but, individual susceptibility and multiple morbility are of major importance. Now a days there are strategies for their prevention and treatment, and, in cases of true actual intolerance or serious adverse events, alternative mechanisms to reduce c-LDL are available. Statins are one of the most widely used drugs in modern medicine and as such, it is important to recognize their adverse events in order to prevent them and to make and early diagnosis and treatment.

Keywords: Statin myopathy, statin toxicity, lipid lowering adverse effects, drug-induced myopathy, statin intolerance.

Desde el punto de vista farmacológico, son inhibidores competitivos de la 3-hidroxi-3-metilglutaril-coenzima A (HMG-CoA) reductasa, enzima que participa en la síntesis de colesterol, la cual, además de reducir el c-LDL, le han sido descritas acciones en diferentes tejidos y en diversas enfermedades, lo que se ha denominado como efectos pleiotrópicos de las estatinas (del griego pleíon $(\pi \lambda \varepsilon i ́ \omega v)$ : "muchos" o "más" y trópos: cambios). ${ }^{1}$

Las estatinas corresponden al grupo de fármacos más prescritos en todo el mundo; en 2014 en Estados Unidos de América, por ejemplo, Pencina y colaboradores estimaron que, por lo menos, 56 millones de estadounidenses tomarían estatinas si cumplieran las recomendaciones del Colegio Americano de Cardiología y de la Asociación Americana del Corazón. ${ }^{3}$ En la República mexicana, de acuerdo con la última Encuesta Nacional de Salud 2018 (ENSANUT 2018), 17.7\% de los adultos mayores de 20 años tiene cifras elevadas de lípidos, ${ }^{4}$ lo que los convierte en usuarios potenciales de medicamentos hipolipemiantes. Pese a que se han publicado un número significativo de reacciones adversas por el empleo de estatinas, algunas requieren de estudios para su mejor caracterización y otras no tienen repercusiones importantes; los daños más graves 
y frecuentes, son la toxicidad muscular y la hepática. Se sabe que poco después de que se generalizó el empleo de las estatinas, se publicaron los primeros pacientes con toxicidad muscular; sin embargo, no se le dio la suficiente importancia, hasta que en 2001 se informó de casos de rabdomiólisis asociada con la ingesta de cerivastatina. Posteriormente, se publicaron más casos, los cuales se relacionaron con la ingesta concomitante de lovastatina y gemfibrozil. Estas graves complicaciones propiciaron la suspensión de la cerivastatina del mercado en el mundo. 5,6

En los últimos años, los informes de toxicidad por estatinas se han enfocado a entidades aisladas, por ejemplo, miopatía, mialgias, rabdomiólisis e intolerancia a la droga. Por esta razón, el propósito de este escrito es actualizar la revisión del daño muscular por estatinas.

\section{NOMENCLATURA}

Uno de los problemas existente en la miopatía por estatinas es el relacionado con su definición, puesto que la palabra miopatía es un término muy general, que en los diccionarios en idioma inglés alude a cualquier enfermedad del músculo. En los diccionarios médicos en castellano, la refieren de la misma manera, y — como ocurre con la mayoría de la nomenclatura científico-tecnológica- el término no aparece en el Diccionario de la Lengua Española de la RAE. Esto ha dificultado su clasificación y también su detección. Se han publicado varias clasificaciones que definen o clasifican el daño muscular relacionado con las estatinas. Es por ello que en la Tabla 1 se anota la propuesta del Colegio Americano de Cardiología, de la Asociación Americana del Corazón y del Instituto Nacional del Corazón, Pulmón y Sangre (ACC/AHA/NHLBI) (la tabla se modificó de acuerdo con los nuevos criterios de la Asociación Nacional de Lípidos de Estados Unidos)..$^{7-9}$

Por la poca precisión de su taxonomía, así como por lo inespecífico de los síntomas, se ha agregado otro concepto denominado "intolerancia a las estatinas", el cual se define como la incapacidad de tolerar dosis bajas, por lo menos, de dos estatinas diferentes, e incluye de manera independiente a los síntomas y a la aparición de biomarcadores anormales que no tengan otra explicación. ${ }^{9,10}$ La intolerancia puede ser completa si no se tolera ningún tipo de estatinas, y parcial o incompleta cuando los síntomas desparecen al disminuir la dosis o al cambio de estatina. Sin embargo, no está muy claro si este nuevo concepto está simplificando o haciendo más compleja la interpretación de los efectos adversos de esta clase de medicamentos.

\section{EPIDEMIOLOGÍA}

La frecuencia real de los efectos adversos de las estatinas aún se desconoce. Esto obedece a problemas en su identificación y a la ausencia de uniformidad en los criterios de diagnóstico, pero también a las diferencias metodológicas en los estudios realizados y a la sobrepercepción derivada del efecto nocebo. ${ }^{11,12}$ Por ejemplo, en algunas investigaciones con controles no muy estrictos, se han informado frecuencias de 30\%; en estudios observacionales, de 10 a $15 \%$, y en estudios controlados, de 1.5 a $5 \% .{ }^{11-13}$ Los efectos adversos musculares en la gran mayoría de los casos se presentan cuando se asocian con medicamentos o condiciones que favorecen su toxicidad, como se anota en la Tabla 2. La ocurrencia de rabdomiólisis se ha informado entre 1 y 1.6 por 100,000 pacientes tratados por año. ${ }^{14}$

\section{PATOGENIA}

La patogenia completa de la toxicidad se desconoce, aunque se han postulado una serie de mecanismos sustentados en múltiples estudios observacionales y experimentales. Los mecanismos podrían ser varios e, incluso, diferentes para las diversas estatinas.

Para su mejor comprensión, en la Figura 1 se representa cómo las estatinas, al inhibir la HMG-CoA reductasa, disminuyen la producción de mevalonato, lo que desencadena una serie de reacciones químicas que culminan con la disminución del colesterol, así como de proteínas isopreniladas y de ubiquinona.

Tabla 1: Alteraciones musculares provocadas por estatinas.

Tipo Características

Mialgias

Miopatía

Miositis

Mionecrosis

Rabdomiólisis
Además del dolor muscular, se incluye el dolor a la palpación de los músculos, rigidez y calambres después del ejercicio Debilidad muscular no relacionada con las mialgias Inflamación muscular diagnosticada por biopsia o resonancia magnética nuclear Elevación de creatincinasa. Leve (más de tres veces), moderada ( $>10$ veces) y grave ( $>50$ veces) Mionecrosis, mioglobinuria y elevación de creatinina (>0.5 mg/dL) 
Tabla 2: Condiciones que aumentan el riesgo de toxicidad por estatinas.

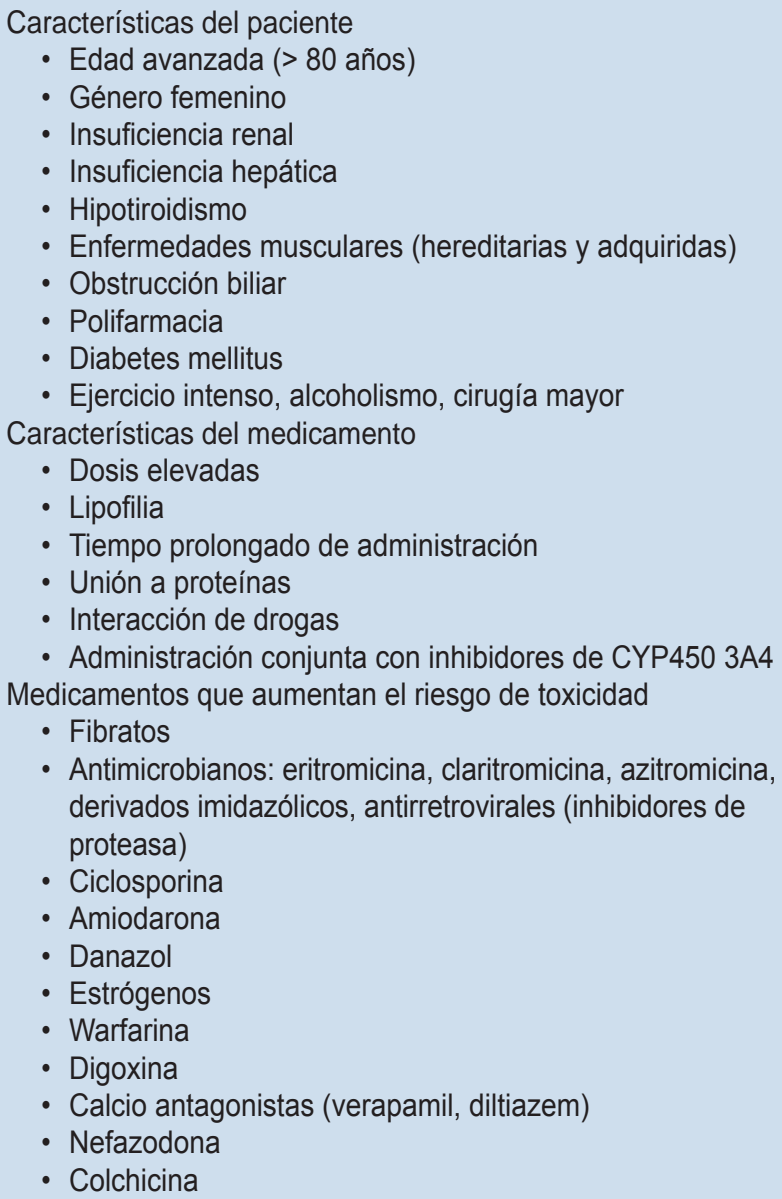
derivados imidazólicos, antirretrovirales (inhibidores de proteasa)

- Ciclosporina

- Amiodarona

- Danazol

- Estrógenos

- Warfarina

- Digoxina

- Calcio antagonistas (verapamil, diltiazem)

- Nefazodona

- Colchicina

Los principales mecanismos de daño que se han propuesto son los siguientes:

\section{a) Disminución del colesterol de las membranas celulares}

Se ha postulado que al bloquear la síntesis de colesterol se disminuye también el colesterol de las membranas de las células del músculo esquelético, lo que genera una membrana frágil e inestable, además de que propicia que la célula muscular sea más susceptible a los diferentes estímulos nocivos. ${ }^{15}$

\section{b) Disminución de ubiquinona}

A la ubiquinona también se le conoce como coenzima Q-10. Es un esteroide isoprenoide que participa en el transporte de electrones durante la fosforilación oxidativa en las mitocondrias de los mamíferos; además, se le han encontrado funciones antioxidantes y reguladoras de apoptosis. Se ha demostrado que el tratamiento con estatinas, sobre todo a dosis altas, reduce los niveles circulantes de Q-10; además, se ha encontrado que el cociente lactato-piruvato es mayor en los pacientes que reciben estatinas, lo que podría sugerir disfunción mitocondrial y un cambio hacia el metabolismo anaeróbico. A mayor abundamiento, se han detectado alteraciones estructurales de las mitocondrias en pacientes que están recibiendo estatinas. ${ }^{15-17}$

\section{c) Disminución de farnesil-pirofosfato y geranil-pirofosfato}

Las estatinas bloquean la producción del farnesil y del geranil-pirofosfato, lo cual disminuye la prenilación de pequeñas proteínas de unión como Ras, Rac y Rho; la disminución de estas proteínas incrementa los niveles de calcio citosólico y activa la vía proteolítica de las caspasas, lo que favorece la muerte celular. ${ }^{18,19}$

Figura 1: Metabolismo del colesterol y su inhibición. Modificado de: Thompson PD, Clarkson PM, Rosenson RS. National lipid association statin safety task force muscle safety expert panel. An assessment of statin safety by muscle experts. Am J Cardiol. 2006; 97: 69C-76C

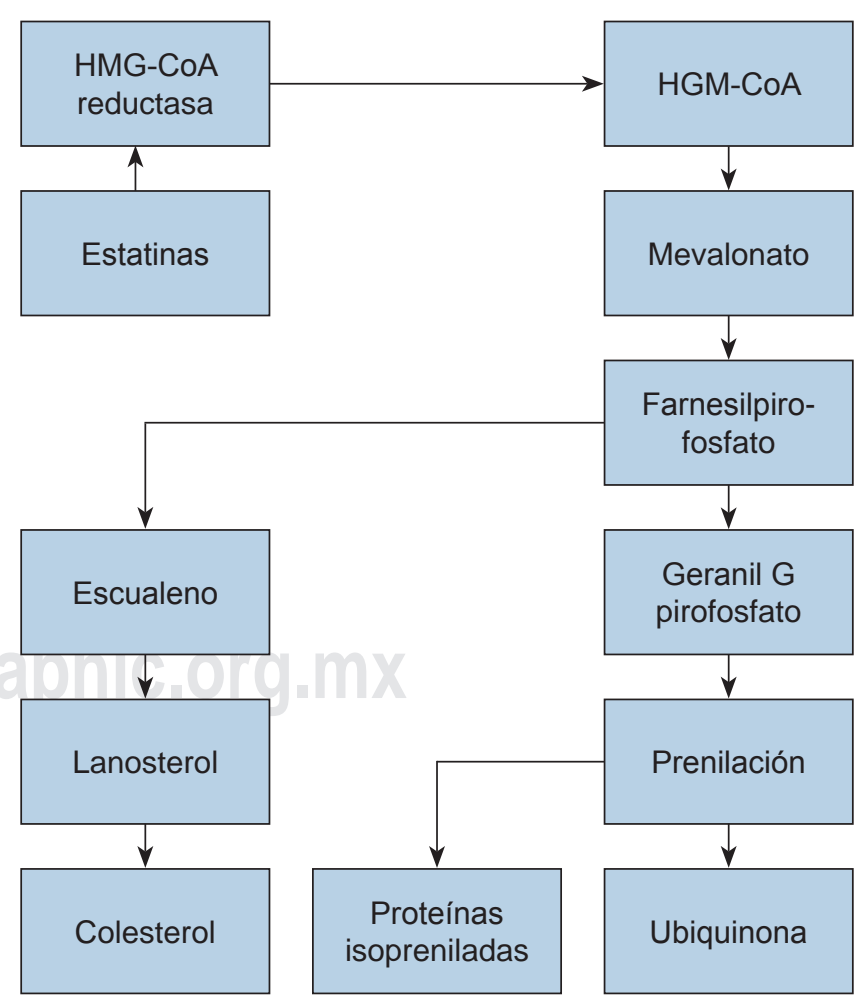




\section{d) Alteración en la expresión de genes: vía ubiquitina-proteasoma}

La masa muscular esquelética es resultado de un complejo mecanismo en donde participan la ruptura y la síntesis de proteínas. En el sistema musculoesquelético, la vía catalítica que rompe y destruye las proteínas miofibrilares, incluyendo la actina y la miosina, es la vía ubiquitinaproteasoma. Se ha postulado que las estatinas pueden afectar la transcripción de genes, lo que ulteriormente favorecería la degradación de proteínas; hay evidencia de que estos medicamentos pueden ser inductores de genes que provocan destrucción muscular y atrofia. Hanai y colaboradores encontraron que después de la administración de lovastatina se expresa el gen atrogin-1 y que, en animales genéticamente modificados, cuando se elimina dicho gen, se puede evitar la toxicidad del fármaco. ${ }^{20}$

\section{e) Fenómenos autoinmunitarios}

Diversos estudios han revelado que las estatinas poseen propiedades inmunomoduladoras, y se ha demostrado que disminuyen la expresión de CD40 en placas aterosclerosas. También, se ha descrito una miopatía necrosante que se asocia con la presencia de anticuerpos contra la HMGCoA, lo que propicia una sobreexposición de los tejidos a las estatinas, incluido, desde luego, al tejido muscular. En estos casos, no siempre existe mejoría al suspender el medicamento, y frecuentemente hay que administrar corticosteroides o inmunodepresores. ${ }^{21-23}$ Además, se considera una variante de miopatía; más adelante, se agregarán otros comentarios.

\section{f) Predisposición genética}

Existe una asociación entre los factores genéticos y la captación hepática de estatinas. Estos medicamentos son transportados dentro de los hepatocitos a través de transporte anióniCo (OATP1B1), el cual está codificado por el gen SLCO1B1. ${ }^{24}$ Se ha propuesto que existen polimorfismos genéticos que favorecen la toxicidad de las estatinas y que se localizan en el gen Co Q2. En un estudio de la red mundial del genoma humano en el que se analizaron 85 sujetos con miopatía por estatinas, se encontró una asociación estadística con un polimorfismo genético localizado en el cromosoma 12.25

$$
\text { g) Lipofilia }
$$

Las estatinas que tienen afinidad por los lípidos penetran fácilmente las células musculares, y se ha evidenciado que las hidrofílicas penetran con menos facilidad a los miocitos. También se requiere de la solubilidad para la salida del medicamento de las células, la cual es favorecida por ciertos polipéptidos como la MRP2 y se ha sugerido que la inhibición de dicha proteína puede favorecer la toxicidad muscular de las estatinas. ${ }^{11,18}$

\section{h) Asociación de condiciones y multimorbilidad}

La multimorbilidad y ciertas condiciones médicas también favorecen la toxicidad muscular de las estatinas (Tabla 2), incluso, algunas de ellas pueden pasar desapercibidas y hacerse patentes cuando reciben el medicamento. El ejemplo más conspicuo lo constituyen las miopatías congénitas subclínicas, las cuales sólo pueden manifestarse cuando se asocian con alguna condición que propicie daño muscular; se desconoce qué porcentaje de miopatías están ubicadas en este grupo. También, habría que tomar en cuenta las fallas orgánicas, las cuales propician un retardo en la eliminación del fármaco, y la polifarmacia, sobre todo cuando coexisten medicamentos que compiten con el metabolismo de las estatinas. Las dosis máximas, denominadas también como de alta intensidad y su administración por tiempo prolongado son condiciones que favorecen claramente el daño muscular. En la Tabla 3 se anotan características farmacológicas de algunas estatinas.

\section{HALLAZGOS HISTOLÓGICOS}

No se han realizado estudios que caractericen a la miopatía por estatinas; su utilidad principal es descartar otro tipo de miopatías; sin embargo, en los casos en los que se han realizado biopsias de músculo, se observan fibras musculares destruidas en "jirones", atrofia muscular, vacuolas de lípidos y ausencia de actividad de la citocromo-oxidasa. ${ }^{17}$

\section{MANIFESTACIONES CLÍNICAS}

No existe un cuadro clínico característico, pero los síntomas musculares son los efectos adversos más frecuentes de las estatinas. Sus manifestaciones varían desde mialgias, fatiga y debilidad leve hasta cuadros graves de rabdomiólisis. Las mialgias son simétricas y bilaterales, por lo general de intensidad moderada, y son más frecuentes en las extremidades inferiores. Los síntomas pueden ser intermitentes y presentarse después de esfuerzo físico, especialmente cuando es vigoroso; por lo general, las manifestaciones aparecen durante el primer mes de haber iniciado el tratamiento. ${ }^{26}$ En ocasiones, sólo es un hallazgo de la exploración física, que se va a manifestar únicamente por debilidad. Se ha informado de pacientes asintomáticos con valores de creatincinasa normales, pero con inflamación muscular en las biopsias. ${ }^{17}$ En la Tabla 1, se señalan los principales fenotipos de los efectos adversos de las estatinas. 
Tabla 3: Características farmacológicas de algunas estatinas.

\begin{tabular}{|c|c|c|c|c|}
\hline Características & Atorvastatina & Pravastatina & Rosuvastatina & Simvastatina \\
\hline Dosis (mg/día) & $10-80$ & $10-40$ & $10-40$ & $10-80$ \\
\hline Vida media (horas) & $15-30$ & 1.3-2.8 & 19 & $2-3$ \\
\hline Solubilidad & Lipofílica & Hidrofílica & Hidrofílica & Lipofílica \\
\hline Metabolismo & СyP3A4 & $\longrightarrow$ & CyP2C9/8 (limitado) & СуР3А4, СуР3А5 \\
\hline Vía de eliminación & Hepática & Hepática y renal & Hepática y renal & Hepática \\
\hline
\end{tabular}

Miopatía autoinmunitaria relacionada con estatinas. Es una variante y complicación rara que se presenta en dos a tres casos por 100 mil usuarios de estatinas. Puede manifestarse al poco tiempo o también después de varios años de haberse iniciado la estatina. Su característica principal es debilidad muscular simétrica y proximal de las extremidades asociada con elevación significativa de creatincinasa. Se encuentran anticuerpos contra la enzima HMG-CoA reductasa, cambios inflamatorios del músculo en la resonancia magnética nuclear y necrosis de miocitos con infiltración de macrófagos en los estudios histológicos. Hay además una expresión importante de HMG-CoA-reductasa en el tejido muscular. Su mecanismo patogénico se desconoce, pero es frecuente en individuos con el alelo DBR1*11:01 del complejo mayor de histocompatibilidad clase II. Se piensa que la unión de la estatina con la HMG-CoA-reductasa genera epítopes desconocidos que van a desencadenar una respuesta autoinmunitaria. El diagnóstico diferencial es con la miopatía inflamatoria autoinmunitaria y con la miositis por cuerpos de inclusión. Pocos pacientes responden exclusivamente a la suspensión de la estatina, y la mayoría requiere tratamiento con inmunodepresores. ${ }^{22,23}$

\section{TRATAMIENTO}

El tratamiento va a depender del tipo de alteración que haya propiciado el medicamento. En general, los síntomas musculares desaparecen totalmente entre dos y tres meses después de la suspensión de la estatina. Para las mialgias, podrían utilizarse analgésicos comunes y, en caso de atrofia muscular, fisioterapia.

1) En pacientes con mionecrosis y rabdomiólisis, debe suspenderse inmediatamente el medicamento. La rabdomiólisis es una condición grave que requiere hospitalización, y su tratamiento escapa a los propósitos de este escrito.
2) Mialgias, miopatía y miositis leve. Aunque también debe suspenderse el fármaco, existen otras conductas, tales como:

a) Investigar el efecto nocebo. El significado real del término alude a los efectos no placenteros, dañinos o indeseables que experimenta un individuo después de recibir algún preparado que carece de efectos farmacológicos, pero el concepto se ha extendido y, actualmente, incluye los síntomas irreales que son percibidos por el paciente cuando éste conoce o ha oído de los efectos secundarios del medicamento. ${ }^{12}$

b) Disminuir la dosis de las estatinas, intentando las dosis más bajas, o bien dosis intermitentes, dos o tres veces por semana; en estos casos, se recomiendan la rosuvastatina o la atorvastatina, que son las estatinas con vidas medias largas; si bien hay que tener presente que estas dosis no han demostrado beneficio en la prevención de eventos cardiovasculares. Si la elevación de CK es mínima o moderada también hay que disminuir la dosis o valorar el cambio de estatina.

c) Cuando hay hipercolesterolemia grave o enfermedad cardiovascular reciente, la mejor opción es la terapia biológica con anticuerpos monoclonales que bloquean la enzima PCSK9 (por sus siglas en inglés: proprotein convertase subtilisin kexin 9). Estos medicamentos actúan bloqueando la degradación de receptores de c-LDL. Para su uso médico, se han aprobado el evolucomab y el alirocumab; actualmente, existen otros que están en las últimas etapas de investigación farmacológica, y otros más que están en proceso de aprobación para su empleo clínico.

d) Otras opciones es agregar ezetimibe o resinas secuestradoras de colesterol, pero el tratamiento es 
complicado y no muy efectivo. Se ha sugerido la administración de CoQ10, pero no existen ensayos clínicos controlados que demuestren su eficacia. Al día de hoy, se encuentra en desarrollo un agente que inhibe una enzima que interviene en la vía del mevalonato (ácido bempedoico); sin embargo, aún está en investigación y todavía no se puede recomendar como una alternativa terapéutica.

Un enfoque recomendable es la prevención y vigilancia de los efectos adversos de las estatinas. Para ello, hay que tener presente sus efectos tóxicos, la multimorbilidad del paciente, solicitar periódicamente creatincinasa, considerar la polifarmacia y evitar en lo posible su prescripción bajo condiciones que favorezcan su toxicidad.

\section{REFERENCIAS}

1. Mehta JL. Statins: understanding clinical use. Philadelphia, USA: Elsevier Inc; 2004.

2. Scandinavian Simvastatin Survival Study Investigators. Randomised trial of cholesterol lowering in 444 patients with coronary heart disease: the Scandinavian Simvastatin Survival Study (4S). Lancet. 1994; 344: 1383-1389.

3. Pencina MJ, Navar-Boggan AM, D'Agostino RB, Williams K, Neely B, Sniderman AD et al. Application of new cholesterol guidelines to a population-based sample. N Engl J Med. 2014; 370: 1422-1431.

4. INSP. Encuesta Nacional de Salud y Nutrición 2018. Disponible en: ensanut.insp.mx.

5. Staffa JA, Chang J, Green L. Cerivastatin and reports of fatal rhabdomyolysis. N Engl J Med. 2002; 346: 539-540.

6. Pierce LR, Wysowski DK, Gross TP. Myopathy and rhabdomyolisis associated with lovastatin-gemfibrozil combination therapy. JAMA. 1990; 264: 71-75.

7. Pasternak RC, Smith SC, Bairey-Merz CN, Grundy SM, Cleeman JI, Lenfant C. ACC/AHA/NHLBI Clinical advisory on the use and safety of statins. JACC. 2002; 40: 567-572.

8. McKenney JM, Davidson MH, Jacobson TA, Guyton JR. Final conclusions and recommendations of the national lipid association statin safety assessment task force. Am J Cardiol. 2006; 97 [Suppl]: 89C-94C.
9. Rosenson RS, Baker S, Jacobson TA, Kopecky SL, Parker BA. An assessment by statin muscle safety task force: 2014 update. J Clin Lipidiol. 2014; 8: S58-S71.

10. Toth PP, Patti AM, Giglio RM, Nikolic D, Castellino G, RizzoM, Banach M. Management of statin intolerance in 2018: still more questions than answers. Am J Cardiovasc Drugs. 2018; 18: 157-173.

11. Ward NC, Watts GF, Eckel RH. Statin toxicity. Mechanistic insights and clinical implications. Cir Res. 2019; 124: 328-350.

12. Ariza AR. El efecto nocebo: los problemas actuales de la información no regulada. Acta Med GA (Mex). 2019; 17: 205-206.

13. Rosenson RS, Baker S, Banache M, Borow KM, Braun LT, Bruckert E et al. Optimizing cholesterol treatment in patients with muscle complaints. J Am Coll Cardiol. 2017; 70: 1290-1301.

14. Thompson PD, Clarkson PM, Rosenson RS. National lipid association statin safety task force muscle safety expert panel. An assessment of statin safety by muscle experts. Am J Cardiol. 2006; 97: 69C-76C.

15. Baker SK, Tarnopolsky. Statin myopathies pathophysiologic and clinical perspectives. Clin Invest Med. 2001; 24: 258-272.

16. Marcoff L, Thompson PD. The role of coenzyme Q10 in statin associated myopathy. A systematic review. J Am Coll Cardiol. 2007; 49: 2231-2237.

17. Phillips PS, Haas RH, Bannykh S, Hathaway S, Nancy RN, Gray L, Kimura BJ et al. Statin-Associated myopathy with normal creatine kinase levels. Ann Intern Med. 2002; 137: 581-585.

18. Thompson P, Clarkson P, Karas RH. Statin-Associated myopathy. JAMA. 2003; 289: 1681-1690.

19. Thoth PP, Harper CHR, Jacobson TA. Clinical characterization and molecular mechanisms of statin myopathy. Expert Rev Cardiovasc Ther. 2008; 6: 955-999.

20. Hanai JI, Cao P, Tanksale P, Imamura S, Koshimizu E, Zhao J et al. The muscle-specific ubiquitin ligase atrogin-1/MAFbx mediates statininduced muscle toxicity. J Clin Invest. 2207; 117: 3940-3951.

21. Needham M, Fabian V, Knezevic W, Panegyres P, Zilko P, Mastaglia FL. Progressive myopathy with up-regulation of MHC-I associated with statin therapy. Neuromuscul Disord. 2007; 17 (2): 194-200.

22. Mammen AL. Statin-associated autoinmune myopathy. N Engl J Med. 2016; 374: 664-669.

23. Nazir S, Lohani S, Tachamo N, Poudel D, Donato A. Statin-associated autoimmune myopathy. A systematic review of 100 cases. J Clin Rheumatol. 2017; 23: 149-153.

24. Gatak A, Faheem O, Thompson PD. The genetics of statin-induced myopathy. Aterosclerosis. 2010; 210: 337-343.

25. The SEARCH Collaborative Group: SLC01B1 variants and statin induced myopathy-A genomewide study. N Engl J Med. 2008; 359: 789-799.

26. Kansen KE, Hildebrand JP, Ferguson EE, Stein JH. Outcomes in patients with statin-associated myopathy. Arch Intern Med. 2005; 165: 2. 